\title{
Audio Effects in the Process of Computer-Aided Processing of Music Signals
}

\author{
Sergei Filatov-Beckmann \\ Russian State Specialized Academy of Arts \\ Moscow State Tchaikovsky's Conservatory \\ Moscow, Russia \\ E-mail: mserg1958@mail.ru
}

\begin{abstract}
The article represents some results of computeraided modeling of music signals, which are generated by means of Sound Forge audio-editing software. Probabilistic measure of information in the form of generated signals is estimated.
\end{abstract}

Keywords-music signal; process; effect; sampling frequency; quantization depth; probabilistic measure of information

\section{INTRODUCTION}

An extensive range of processes and effects that influence the character of music signals is contained in audio-editing software (e.g. Sonar) and virtual-studio software (Cubase, Nuendo, etc.), which enable to process the information represented in various music formats $[1,5]$.

Sound Forge is a relatively simple processing system which allows to conduct acoustic experiments based on modern digital music acoustics and music informatics [1]. Thus, a built-in synthesizer generates the sound based on the so-called signal frequency modulation, providing the following possible forms of signals: SIN; SIN ABS or previous signal module; SQUARE; TRIANGLE; SAW. The above waveforms (except for triangular impulse) are shown in "Fig. 1".
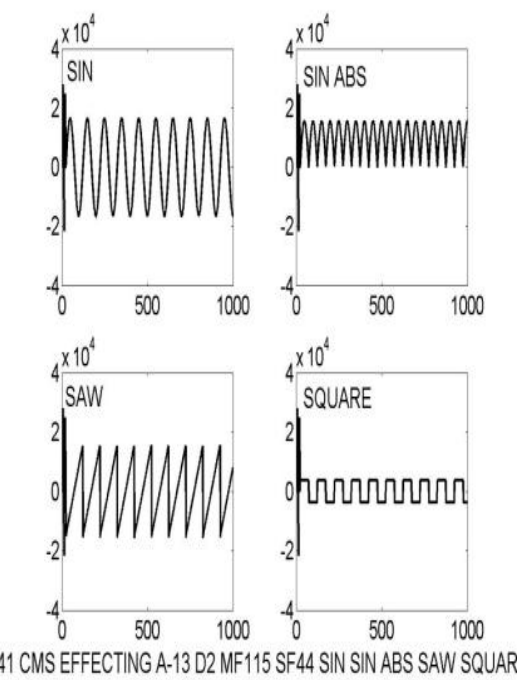

Fig. 1. SIN, SIN ABS, SAW, and SQUARE signal forms
In addition, specific sound effects are generated: BROUN NOISE, FILTERED NOISE and PINK NOISE. These effects are shown in "Fig. 2".

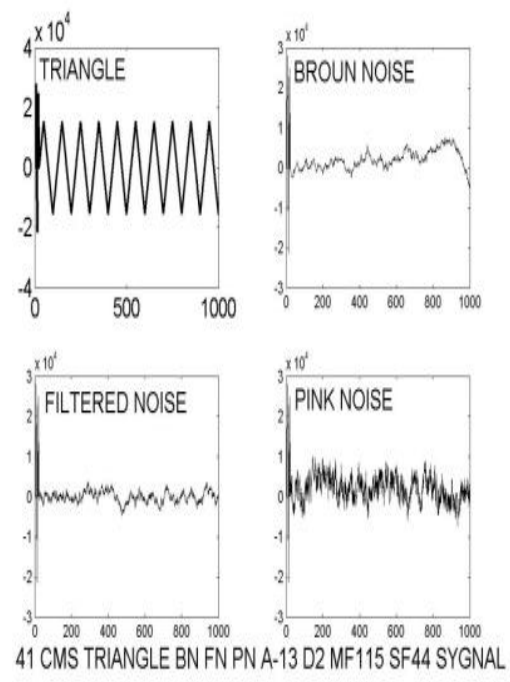

Fig. 2. The forms of TRIANGLE signal and noise effects

\section{WhITE NOISE: COMPUTER RESEARCH}

Another noise effect is WHITE NOISE. It is well known that white-noise spectrum has a uniform distribution stemming from non-correlation (independence) of frequency components of this kind of noise [6].

Any signal fixed (or synthesized) using a computer has two important characteristics. The first one determines the number of counts per unit of time, which generate a signal, and it is called 'Sampling Frequency' $(S F)$. The second one determines the number of levels, which are necessary to reproduce the amplitude (volume) of a signal, and it is called 'Quantization Depth' (D). Thus, each of these signals is represented by an aggregate of count points, and therefore it has a discrete (quantum) nature.

Sound Forge editor allows to modify the characteristics of $S F$ and $D$ in a fairly wide range. The purpose of the series of experiments conducted by us was to assess the impact of 
the variability as to sampling frequency and record depth on basic characteristics of white noise.

Experiments were conducted based on a computer-aided model of music statistics as developed by the author [7]. This model allows to investigate some properties of random music signals by calculating a number of signal characteristics. Some of the characteristics are purely statistical in nature, but more complex dependencies are used as well, borrowed from the toolkit of modern nonlinear dynamics [3]. Statistical variables reflect individual aspects of signal evolution in time, and that allows to obtain a number of roundup characteristics for a signal under consideration. One of the most important characteristics of the kind is probabilistic measure of information introduced by Shannon [4].

The calculation of probabilistic measure of information (m) is made based on the following formula:

$$
\mathrm{m}=-\Sigma \log _{2}(\mathrm{p}) * \mathrm{p},
$$

which represents a finite-difference approximation of a mathematical expectation for a logarithmic function. The use of binary logarithm allows to express the result in the socalled binary units (or bits). The value $p$ in our experiments corresponds to the probability of a particular value of pulsation, arising in the course of generating computer-based signal (the so-called 'Digitization').

Figure 3 shows the values of probabilistic measures of information obtained for the three values of record depth $(D)$ (1, 2 and 3 bytes), and for the four values of sampling frequency $(S F)(2,11,44$ and $96 \mathrm{kHz})$. Value $D$ is laid off on vertical axis, and value $S F-$ on horizontal axis. The availability of a number of values for probabilistic measure allows to build up the spatial distribution (or geometry) of this measure represented as a field with drawn lines of equal values (in bits):

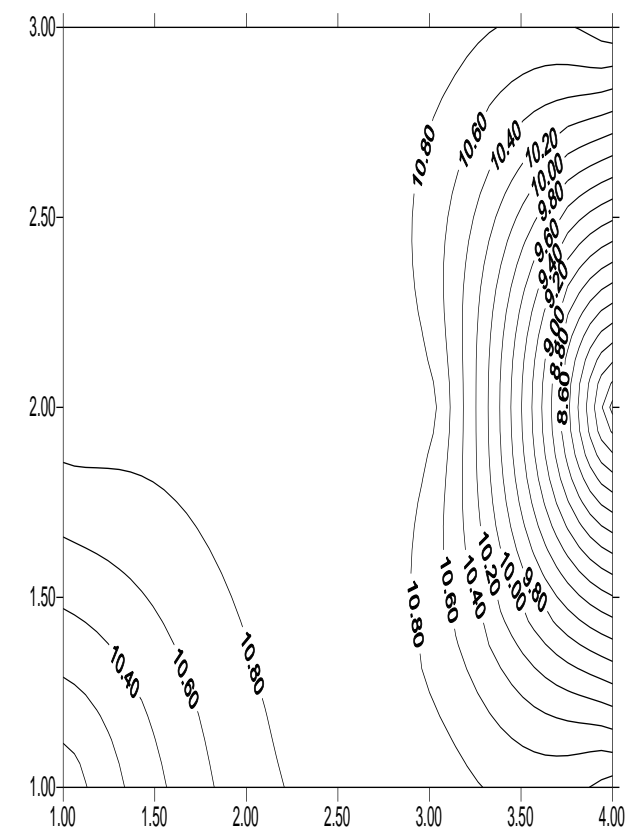

Fig. 3. Geometry of probabilistic measure of information for white noise (bits)
The results of experiments show that reduced values $S F$ and $D$ lead to the reduction of signal activity (the lower left corner of the figure). The reduction of probabilistic measure $(m)$ in the right part of the figure (by 2 bits) may be attributed to a very complex (needle-type) nature of the pulsation of a signal under consideration. However, the field of $m$ value in general is quite homogeneous and lowsensitive to the variations of $S F$ and $D$.

Figure 4 shows a field of probabilistic measure of information obtained for a micro-interval with a value of 20 cents built up around frequency standard of $415.3 \mathrm{~Hz}$. This standard belongs to Garbuzov's Tuple, statistics compiled by N.A. Garbuzov, prominent Russian music thinker, in the course of studying the phenomenon of Zoning of absolute hearing [2]:

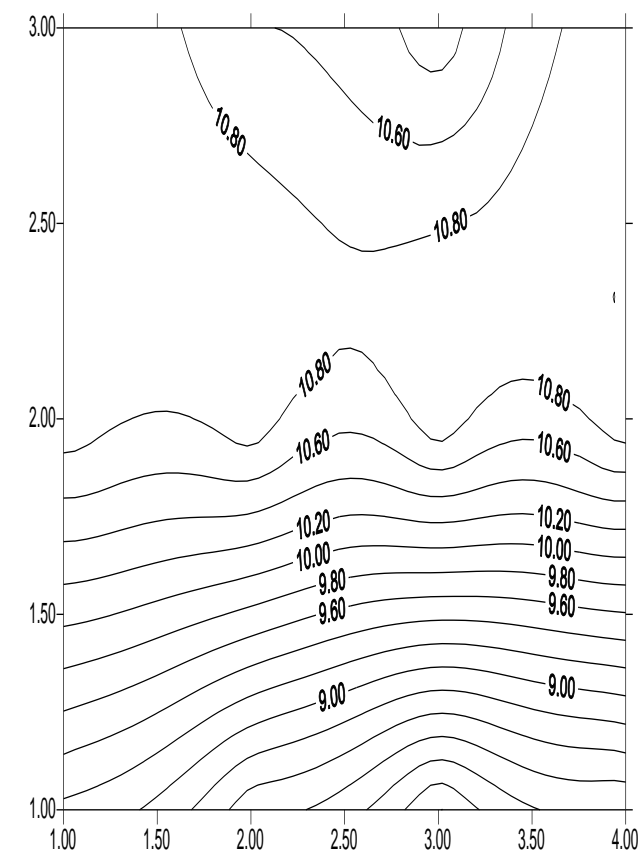

Fig. 4. Topology of probabilistic measure of information for a microinterval of 20 cents, Garbuzov's Tuple (bits)

The field obtained has greater variability as compared to previous figure. However, in this case the maximum of information (about 11 bits) corresponds to record depth equal to two bytes and preserves invariability as to sampling frequency.

\section{RESULTS}

It could be assumed that the indicated effect is associated with the peculiarities of signal perception by human audio system. In other words, practices of sound recording and sound design lead precisely to those very parameters of sound recording which are optimal. How could this be explained?

Let us imagine the ratio of values for record depth and sampling frequency. Let us assume that record depth is two bytes, which gives us a number of 65.536 levels (quantization); in this case, sample frequencies (11.025 to $96.000 \mathrm{~Hz})$ appear to be identical in terms of the order of 
values for quantization depth. Indeed, 11.025, 22.050, 48.000, and 96.000 are close, in a sense, to number 65.536.

Let us assume that the record depth is one byte. In this case, we get 256 record levels, while sampling frequency belongs to the interval of $11.025-96.000 \mathrm{~Hz}$. It is clear enough that it is hard to compare such numbers, as they have different order.

Let us assume that the record depth is three bytes. In this case, we get about 17 million quantization levels; in turn, is hard compare such a number to sampling frequencies (11.025-96.000).

Let us compare the results obtained. In case of record depth equal to one byte, we get some rectangle, the base of which is much larger than its height, i.e. almost onedimensional figure. In case of record depth equal to three bytes, we get a rectangle the base of which is far less than its height, and again, that generates one-dimensional function (almost Dirac delta function).

It could be supposed that audio system of human perception selects a particular optimal range of parameters for the digitization of music signals, which appears to be close to the sampling frequency of $48.000 \mathrm{~Hz}$ and the record depth of 2 bytes. Hopefully, the results obtained open a prospect for further research in the field of music psychoacoustics, and they may be useful for the experts engaged in the field of music sound design.

\section{REFERENCES}

[1] Aldoshina I., Pritts R. Music Acoustics. Textbook. - St. Petersburg: Compozitor. St. Petersburg, 2006. - 720 pages: illustrated.

[2] N. A. Garbuzov -musician, researcher, educator: A collection of articles / Compiled by O. Sakhaltuyeva, O. Sokolova. - Moscow: Muzyka, 1980.

[3] Malinetsky G.G., Potapov A.B. Modern Problems of Non-Linear Dynamics. - Moscow: Editorial URSS, 2000. - 336 pages.

[4] Fundamentals of Cybernetics. Theory of Cybernetic Systems. Edited by K.A. Pupkov. A Study letter for universities. Moscow: Vysshaya Shkola, 1976. - 408 pages: illustrated.

[5] Petelin R.Y., Petelin Y.V. Cubase SX. The Secrets of the Craft. - St. Petersburg: BKV-Peterburg, 2003. - 640 pages: illustrated.

[6] Sergiyenko A. Digital Signal Processing: A Textbook for Higher Education Institutions. 2nd Edition. - St. Petersburg: Piter. 2007. 751 pages: illustrated.

[7] Filatov-Beckmann S.A. Computer-Aided Music Modeling: A Textbook for Higher Education Institutions. - Moscow: Sam Poligrafist, OJSC, 2015. 160 pages: illustrated.

[8] Tsoller S.A. PC-Based Music Creation: From Simple to Complicated. - St. Petersburg: BKV-Peterburg, 2005. - 320 pages: illustrated. 